\title{
Calculation of the $Z+$ jet cross section including transverse momenta of initial partons
}

\author{
M. Deak, ${ }^{1}$ A. van Hameren, ${ }^{1}$ H. Jung, ${ }^{2}$ A. Kusina, ${ }^{1}$ K. Kutak,,${ }^{1,4}$ and M. Serino ${ }^{3}$ \\ ${ }^{1}$ Institute of Nuclear Physics, Polish Academy of Sciences, \\ ulica Radzikowskiego 152, 31-342 Cracow, Poland \\ ${ }^{2}$ DESY, 22603 Hamburg, Germany \\ ${ }^{3}$ Department of Physics, Ben Gurion University of the Negev, Beer Sheva 8410501, Israel \\ ${ }^{4}$ Theoretical Physics Department, CERN, 1211 Geneva 23, Switzerland
}

(Received 7 April 2019; published 10 May 2019)

\begin{abstract}
We perform calculations of $Z+$ jet cross section taking into account the transverse momenta of the initial partons. Transverse momentum dependent (TMD) parton densities obtained with the parton branching method are used and higher order corrections are included via TMD parton showers in the initial state. The predictions are compared to measurements of forward $Z+$ jet production of the $\mathrm{LHCb}$ Collaboration at $\sqrt{s}=7 \mathrm{TeV}$. We show that the results obtained in $k_{T}$-factorization are in good agreement with results obtained from a next-to-leading order calculation matched with traditional parton showers. We also demonstrate that in the forward rapidity region, $k_{T}$-factorization and hybrid factorization predictions agree with each other.
\end{abstract}

DOI: 10.1103/PhysRevD.99.094011

\section{INTRODUCTION}

The Large Hadron Collider opened opportunities to explore kinematic regions where particles produced in high-energy collisions possess large transverse momenta and a wide range of available rapidities. The production of electroweak bosons and jets is a vital test of the Standard Model. Furthermore, studies of the associated production of electroweak bosons and jets provide important insights into the transverse partonic structure of hadrons. In particular, motivated by earlier studies [1-5], one can use recent theoretical and technical advancements to study in more detail transverse momentum dependent (TMD) parton densities [6].

Final states of the $Z+$ jet type, being a combination of colored and colorless partons and particles, give the opportunity for investigations which complement the results obtained in studies of pure jet final states [7]. This is because final state rescatterings due to soft color exchanges have less impact on the properties of the produced final state as compared to pure jet final states.

This work focuses on predictions using $k_{T}$-factorization [8] (also referred to as high-energy factorization) as implemented in the parton-level event generator KaTie [9]

Published by the American Physical Society under the terms of the Creative Commons Attribution 4.0 International license. Further distribution of this work must maintain attribution to the author(s) and the published article's title, journal citation, and DOI. Funded by SCOAP ${ }^{3}$. combined with a TMD initial state parton shower implementation in new version of CASCADE $[10,11]$ compared to $Z+$ jet measurements of the LHCb Collaboration [13].

The $k_{T}$-factorization formula for the inclusive cross section schematically reads

$\sigma=\int \frac{1}{F} d P S \sum_{i, j} \mathcal{A}_{i}\left(x_{1}, k_{T 1}, \mu_{F}\right) \otimes|M E|^{2} \otimes \mathcal{A}_{j}\left(x_{2}, k_{T 2}, \mu_{F}\right)$,

where $F$ is the flux, $P S$ is the final state phase space, and $M E$ is the partonic matrix element. The TMD parton distributions $\mathcal{A}_{i}\left(x, k_{T}, \mu_{F}\right)$ depend for a parton of type $i$ on the longitudinal momentum fraction $x$, the factorization scale $\mu_{F}$, and the transverse momentum $k_{T}$, i.e., the momentum perpendicular to the beam collision axis. The formula is valid when $x$ is not too small and additional effects from gluon recombinations can be neglected $[14,15]$. The matrix elements in the formula above are efficiently calculated numerically using helicity methods [16,17] and recursion relation as implemented in KaTie [9], giving the same results as using Lipatov's effective action [18].

The TMD parton densities can be defined by introducing operators whose expectation values count the number of partons and their evolution is given by renormalization of divergences either in rapidity or transversal momentum. One can also construct TMD parton densities starting from the collinear parton densities by "unfolding" them by the Watt-Kimber-Martin-Ryskin prescription $[19,20]$. In the 
parton branching (PB) method [21,22], which will be used in this article, the TMD parton density is constructed by a parton branching algorithm to solve the DokshitzerGribov-Lipatov-Altarelli-Parisi evolution equations. The starting distributions are obtained from a fit to inclusive deep inelastic scattering cross section measurements [23], using next-to-leading order (NLO) splitting functions. The advantage of using PB TMD parton densities is that once integrated over the transverse momentum the collinear parton density functions are obtained, which is essential for a consistent comparison between $k_{T}$-factorization and collinear approaches. The PB TMD parton densities are well suited to construct initial state parton showers [11] in such a way that the kinematics of the hard process are fixed and no kinematic corrections are applied when the parton shower is added, as opposed to what is typically done in genuine collinear physics based Monte Carlo generators. With the PB TMD parton densities one can address observables initiated by initial state off-shell quarks and, in the end, perform a Monte Carlo simulation of the whole event, e.g., for a process

$$
P_{A}+P_{B} \rightarrow Z / \gamma^{*}+j+X \rightarrow \mu^{+} \mu^{-}+j+X,
$$

where $P_{A}$ and $P_{B}$ are the colliding protons which produce the intermediate state with one jet $j$ and one $Z / \gamma^{*}$ boson and other unobserved particles in a state $X$. We compare the prediction with a measurement obtained by LHCb [13], where the Z-boson decay into muon pairs was studied.

\section{RESULTS}

We present results computed in the framework of $k_{T^{-}}$ factorization and hybrid factorization [7,12] and compare them to the recent $Z+$ jet measurements of LHCb [13]. We use KaTie [9] in order to produce parton-level events with off-shell initial state momenta. These events are then further processed by CASCADE [10] with its extension for a complete initial state parton shower for all flavors, as described in Ref. [11]. The final state parton shower and hadronization are performed by PYTHIA [24]. We also use calculations performed in collinear factorization, with parton showers added, in leading order (LO) for $Z+1$ jet and at NLO for $Z+2$ jets using POWHEG [25] together with PYTHIA [26] for parton showering, multiparton interaction, and hadronization. For all calculations we use two-loop $\alpha_{s}$ with $\alpha_{s}\left(m_{Z}\right)=0.118$. The parton branching TMD parton densities [23] (more specifically PB-NLO2018-Set 2), available in TMDlib [27], are used for $k_{T}$ dependent calculations while for the hybrid approach (with one on-shell and one off-shell initial parton) and the collinear approach we use the HERAPDF20_NLO_EIG collinear parton density function (PDFs) [28]. The PB-NLO-2018 TMD parton densities, once integrated over $k_{T}$, reproduce HERAPDF20_NLO, by construction.
The analysis of the final state particles is performed with the RIVET framework [29].

From all the distributions measured by LHCb [13] we will concentrate on the $\Delta \phi$ and $p_{T}^{Z}$ distributions, for which going beyond the collinear approximation is most relevant.

\section{A. Parton-level results}

We start by discussing results of calculations performed at the parton level (without any showering or hadronization, but with the decay $Z / \gamma^{*} \rightarrow \mu^{+} \mu^{-}$), by convolving the TMD densities with the matrix elements, as schematically described by Eq. (1).

In Fig. 1 we show distributions of $\Delta \phi$ and $p_{T}^{Z}$ calculated using two off-shell initial state partons for two different cuts on the jet transverse momentum (as used by LHCb): $p_{T}^{\text {jet }}>$ $10 \mathrm{GeV}$ and $p_{T}^{\text {jet }}>20 \mathrm{GeV}$. The renormalization and factorization scale was set to $\mu=\sqrt{m_{Z}^{2}+\left(p_{T}^{\text {jet }}\right)^{2}}$, which is varied by a factor of 2 up and down in order to estimate the scale uncertainty. We can see that for both cut choices the description of the $\Delta \phi$ distribution is very good and the data lie within the scale uncertainties. The measurement of $p_{T}^{Z}$ is also well described, with the exception of the lowest $p_{T}$-region, where the data are underestimated. This is the region that is driven by the initial state transverse momentum described by the TMD parton densities. In a collinear parton-level $2 \rightarrow 2$ calculation the region below the $p_{T}^{\text {jet }}$ cut would be empty.

In Fig. 2 we investigate the importance of the scale choice by comparing the calculation with $\mu=\sqrt{m_{Z}^{2}+\left(p_{T}^{\text {jet }}\right)^{2}}$ (Fig. 1) with different choices: $\mu=$ $\left(p_{T}^{\text {jet }}+p_{T}^{Z}+m_{Z}\right) / 3$ and $\mu=\sqrt{\hat{s}+Q_{t}^{2}}$ with $Q_{t}=p_{T}^{\text {initial state }}$. We can see that the scale $\mu=\sqrt{\hat{s}+Q_{t}^{2}}$, which is motivated by angular ordering [30], gives results similar to our original scale choice, while the choice $\mu=\left(p_{T}^{\text {jet }}+p_{T}^{Z}+\right.$ $\left.m_{Z}\right) / 3$ leads to significant differences. However, most of the time the results are still within the scale uncertainty of the results from Fig. 1. Based on this observation, we will use the scale $\mu=\sqrt{m_{Z}^{2}+\left(p_{T}^{\text {jet }}\right)^{2}}$ in the following.

In Fig. 3 we compare the predictions obtained within $k_{T^{-}}$ factorization (presented in Fig. 1) to the corresponding predictions obtained in the hybrid (the lower $x$ initial state parton is off-shell while the higher $x$ initial state parton is on-shell) and collinear approaches at parton level. We can see that for both $\Delta \phi$ and $p_{T}^{Z}$ distributions (with both cut choices) the results of the hybrid approach are very similar to the ones of $k_{T}$-factorization. This shows that the hybrid factorization works well where it is expected to work, i.e., in the forward region where asymmetric values of $x_{1}$ and $x_{2}$ are probed. The key point in this study are the TMD parton densities and collinear PDFs used: the $k_{T}$-integrated $\mathrm{PB}$ TMD parton density is identical to the collinear PDF by 


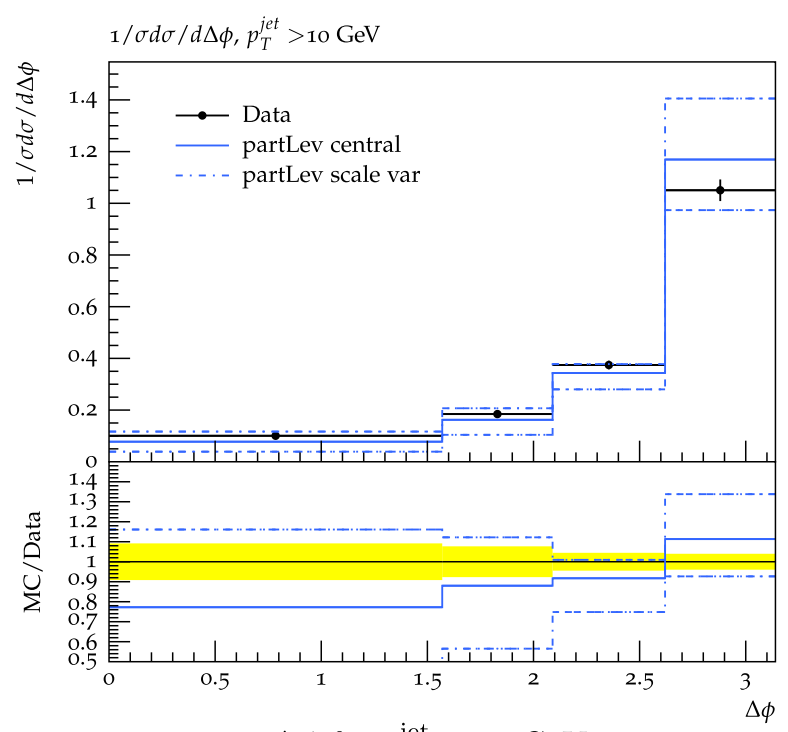

(a) $\Delta \phi$ for $p_{T}^{\text {jet }}>10 \mathrm{GeV}$

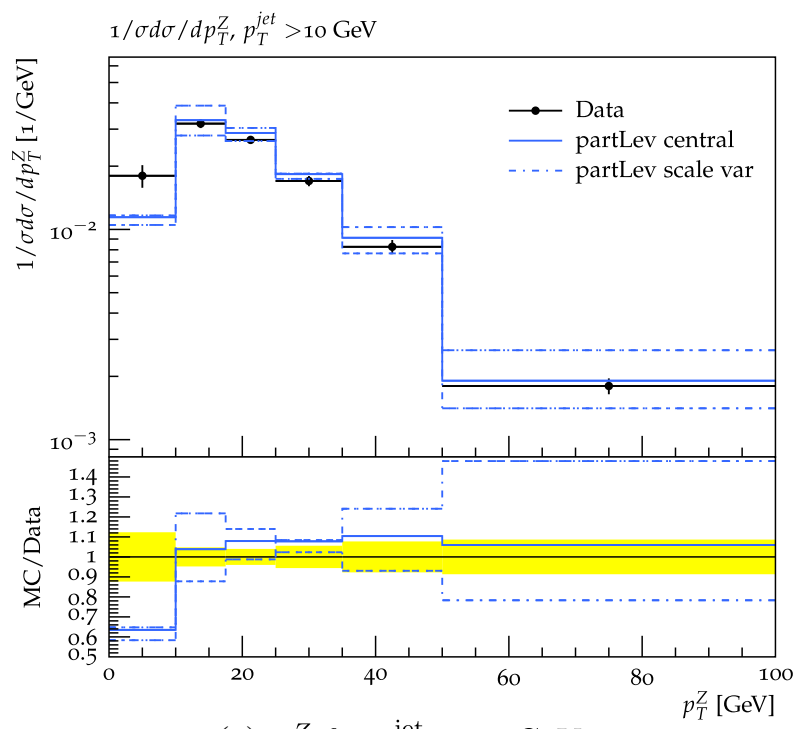

(c) $p_{T}^{Z}$ for $p_{T}^{\text {jet }}>10 \mathrm{GeV}$

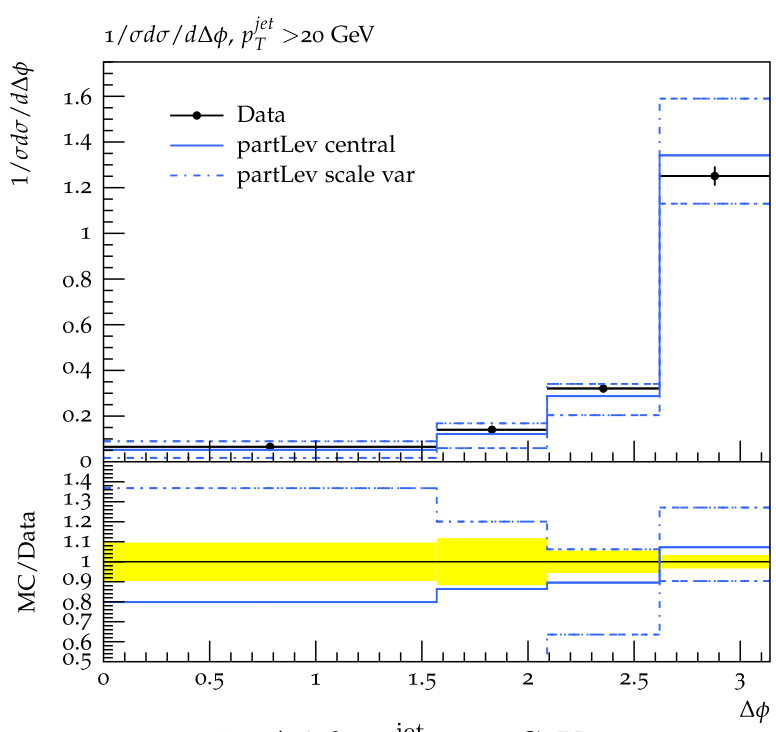

(b) $\Delta \phi$ for $p_{T}^{\text {jet }}>20 \mathrm{GeV}$

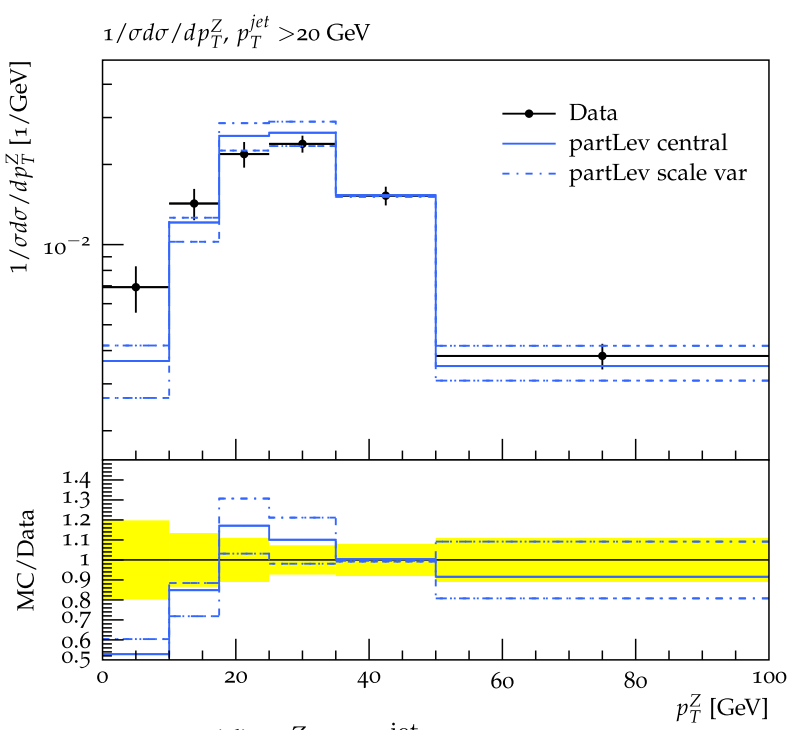

(d) $p_{T}^{Z}$ for $p_{T}^{\text {jet }}>20 \mathrm{GeV}$

FIG. 1. Scale variation for $\Delta \phi$ and $p_{T}^{Z}$ distributions calculated at the parton level (without any showers) using a factorization/ renormalization scale $\mu=\sqrt{m_{Z}^{2}+\left(p_{T}^{\text {jet }}\right)^{2}}$. Comparison to the LHCb measurements [13].

construction. When $k_{T}$-factorization is used in the forward rapidity region, the $k_{T}$ on the large $x$ side is limited and the matrix elements with two off-shell initial state particles effectively become on shell-of shell, and in the process of evaluating the cross section the PDF on that side is integrated to yield a collinear PDF. ${ }^{1}$ In the hybrid factorization calculation, the collinear PDF was used from the beginning. A more detailed analysis of it will be provided

\footnotetext{
${ }^{1}$ The main contribution will be coming from the configuration when gluons feature small $x$ and are off shell, whereas, the quarks have large $x$ and are nearly on shell.
}

in Sec. II C where we discuss correlations of transverse momenta and $x$ values of the initial partons.

In Fig. 3 we additionally present results obtained within collinear factorization. The motivation is to show that a purely collinear approach is not able to describe certain distributions at $\mathrm{LO}$ and higher order corrections are needed. This is especially visible for the $\Delta \phi$ distribution which reduces to a delta function at LO. The cut on the $p_{T}^{\text {jet }}$ restricts also the $p_{T}^{Z}$ distribution since there is no recoil that would allow the $Z$ boson to gain additional transverse momentum. Later we will compare our results also with predictions in collinear factorization at LO but including parton showers as well as with NLO predictions. 


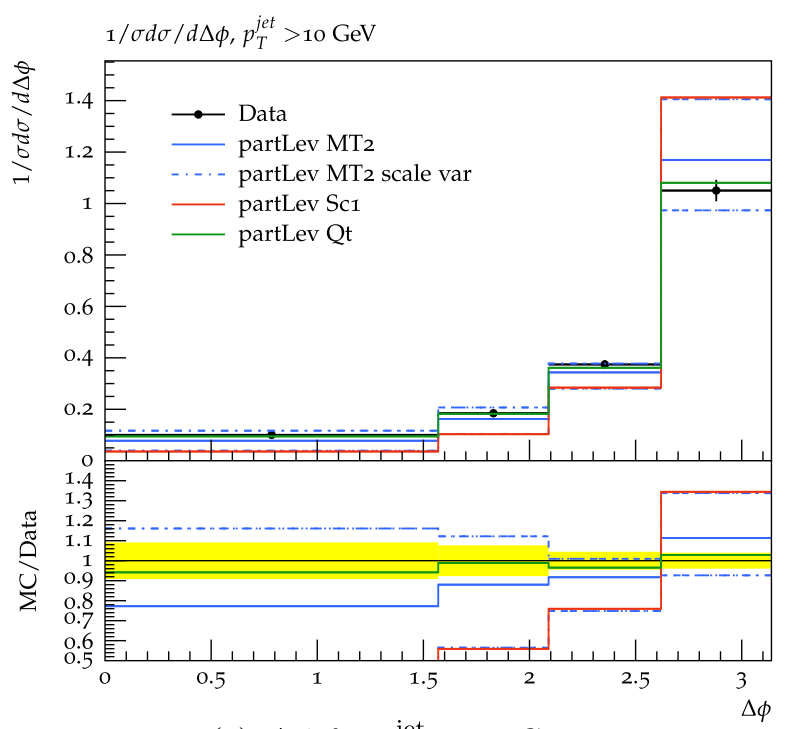

(a) $\Delta \phi$ for $p_{T}^{\text {jet }}>10 \mathrm{GeV}$

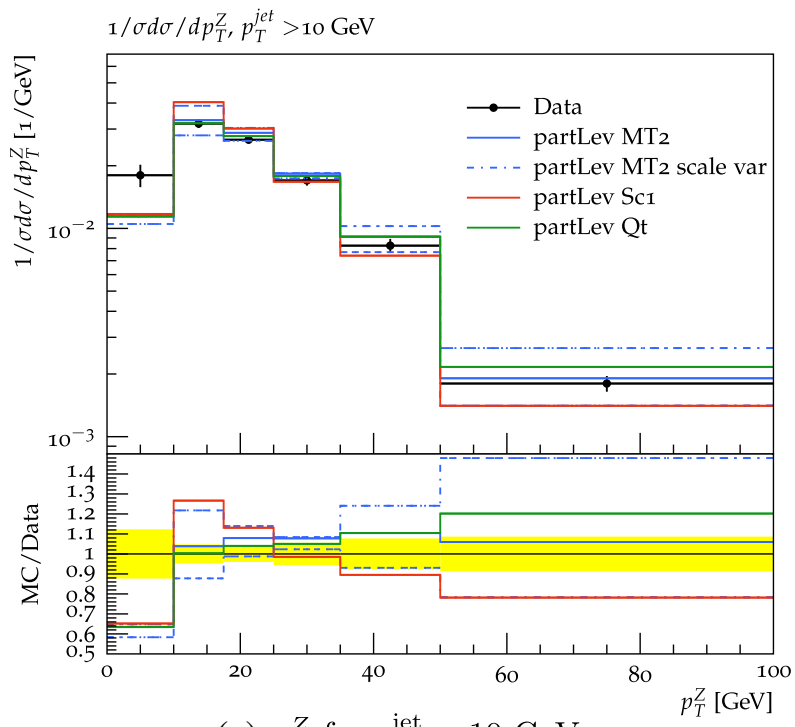

(c) $p_{T}^{Z}$ for $p_{T}^{\text {jet }}>10 \mathrm{GeV}$

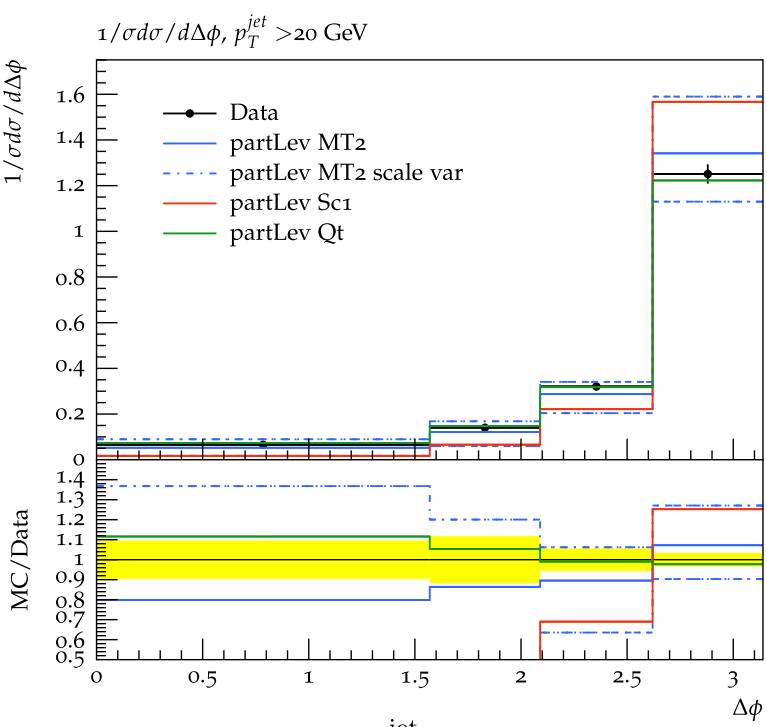

(b) $\Delta \phi$ for $p_{T}^{\text {jet }}>20 \mathrm{GeV}$

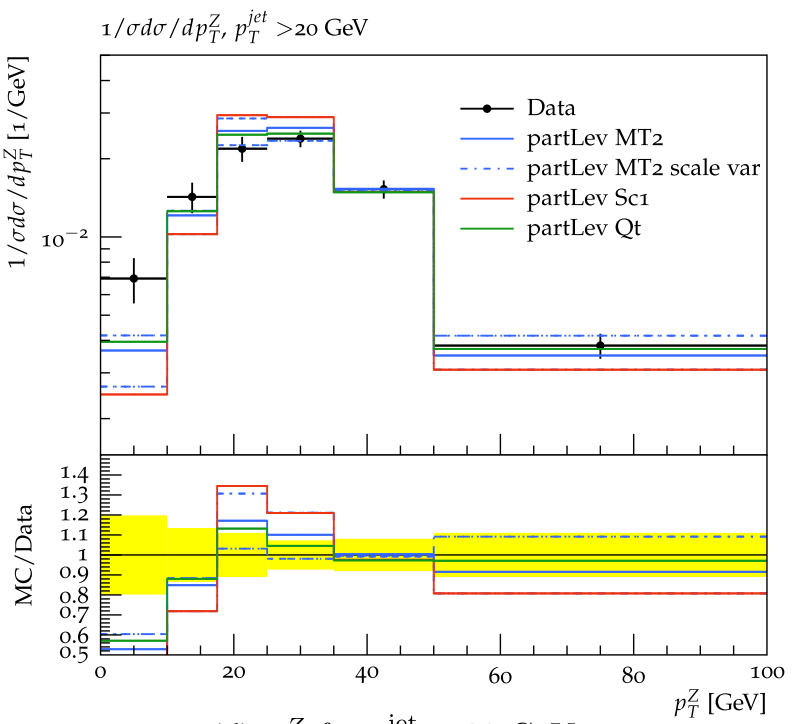

(d) $p_{T}^{Z}$ for $p_{T}^{\text {jet }}>20 \mathrm{GeV}$

FIG. 2. Comparison of different scale choices. 2(a), 2(c) for $p_{T}^{\text {jet }}>10 \mathrm{GeV}$ and 2(b), 2(d) for $p_{T}^{\text {jet }}>20 \mathrm{GeV}:$ MT2: $\mu=\sqrt{m_{Z}^{2}+\left(p_{T}^{\text {jet }}\right)^{2}}$, Sc1: $\left(p_{T}^{\text {jet }}+p_{T}^{Z}+m_{Z}\right) / 3$, Qt: $\sqrt{\hat{s}+Q_{t}^{2}}$ for $\Delta \phi$ and $p_{T}^{Z}$ distributions calculated at the parton level (without any showers). Comparison to the $\mathrm{LHCb}$ measurements [13].

\section{B. Showered results}

In this section we present results using an updated version of the CASCADE Monte Carlo event generator $[10,11,31]$, allowing us to process Les Houches event files [32], generated by KaTie and to provide initial state parton showers according to the PB TMD parton densities. The calculations are also supplemented with standard final state parton shower and hadronization [24], the multiparton interactions are not included. When off-shell matrix elements are used, the transverse momenta of the initial state partons are already included according to the TMD parton densities. In the case of collinear matrix elements, first a transverse momentum is generated according to the TMD and added to the event record in such a way that the mass of the hard process $\hat{s}$ is preserved, and the event is showered, while the parton shower does not change the kinematics of the hard process (after the transverse momentum is included). In hybrid factorization, the initial state parton shower is included only for the off-shell leg, while the other leg stays at $k_{T}=0$. It is important to stress that the parton densities used in the calculations are applied in a consistent way.

In Fig. 4 we compare results obtained using $k_{T^{-}}$ factorization, hybrid factorization, and collinear factorization [using the scale $\mu=\sqrt{m_{Z}^{2}+\left(p_{T}^{\text {jet }}\right)^{2}}$ ]. We can see that, 


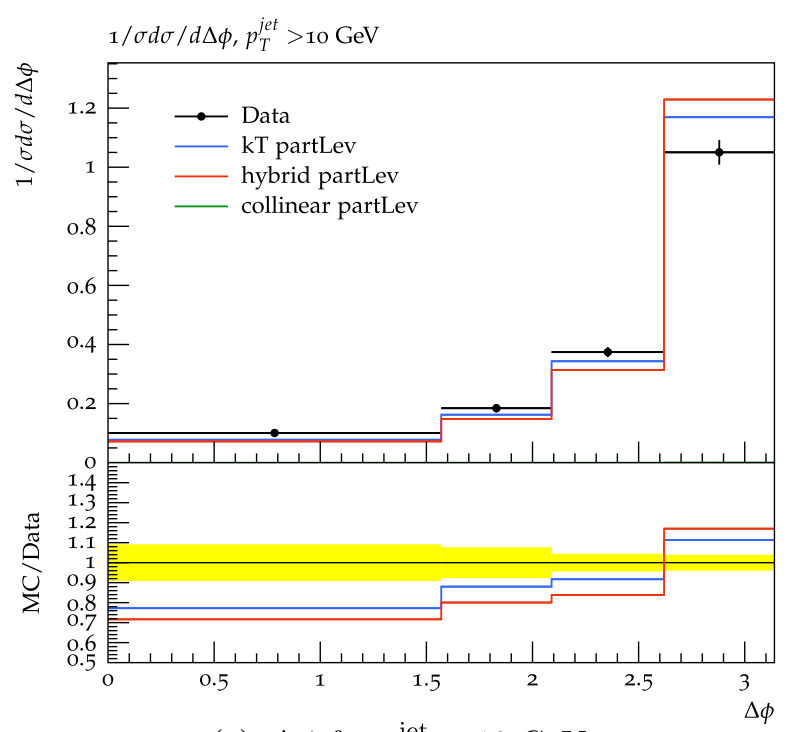

(a) $\Delta \phi$ for $p_{T}^{\text {jet }}>10 \mathrm{GeV}$

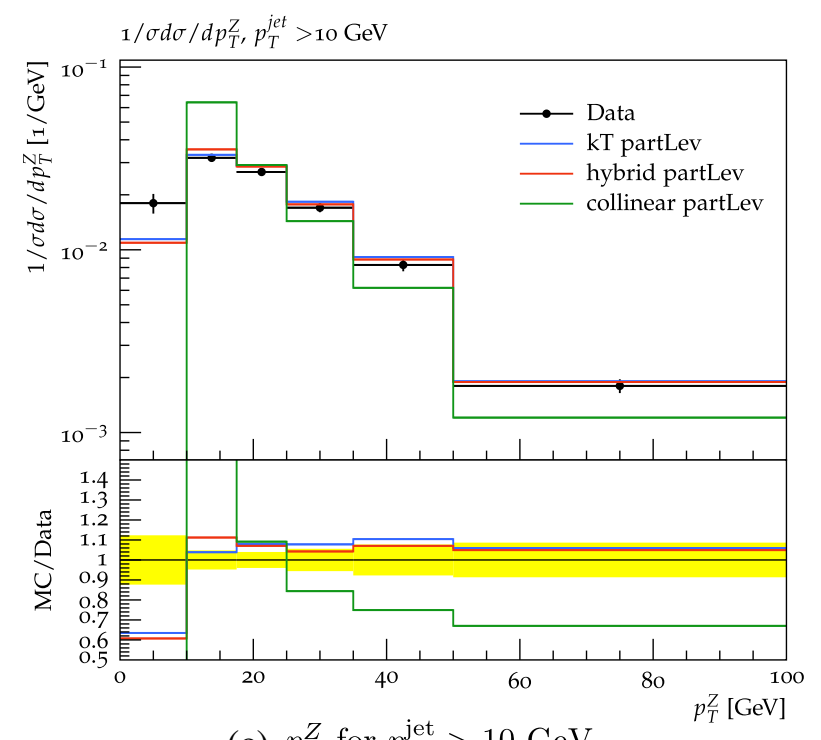

(c) $p_{T}^{Z}$ for $p_{T}^{\text {jet }}>10 \mathrm{GeV}$

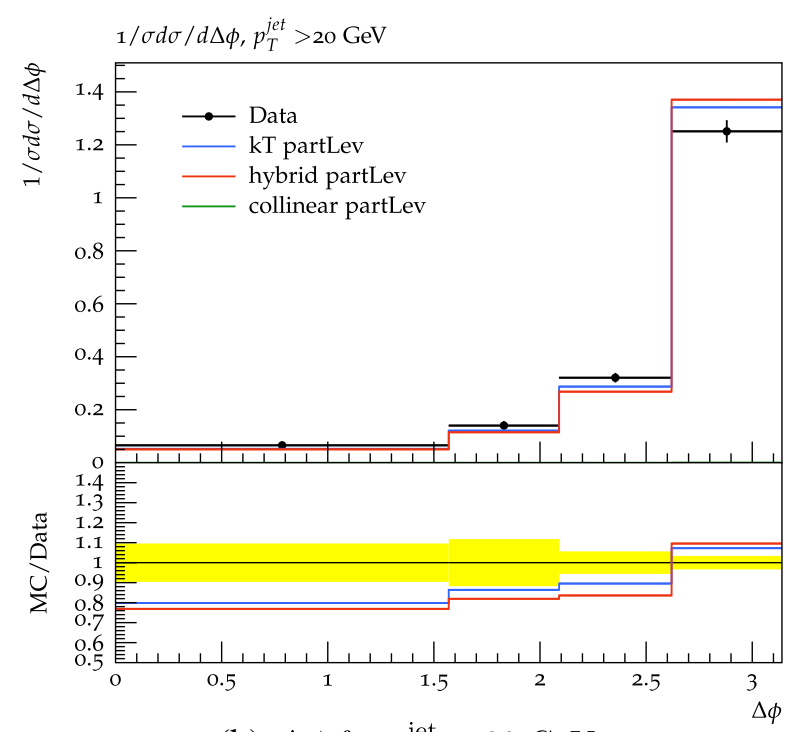

(b) $\Delta \phi$ for $p_{T}^{\text {jet }}>20 \mathrm{GeV}$

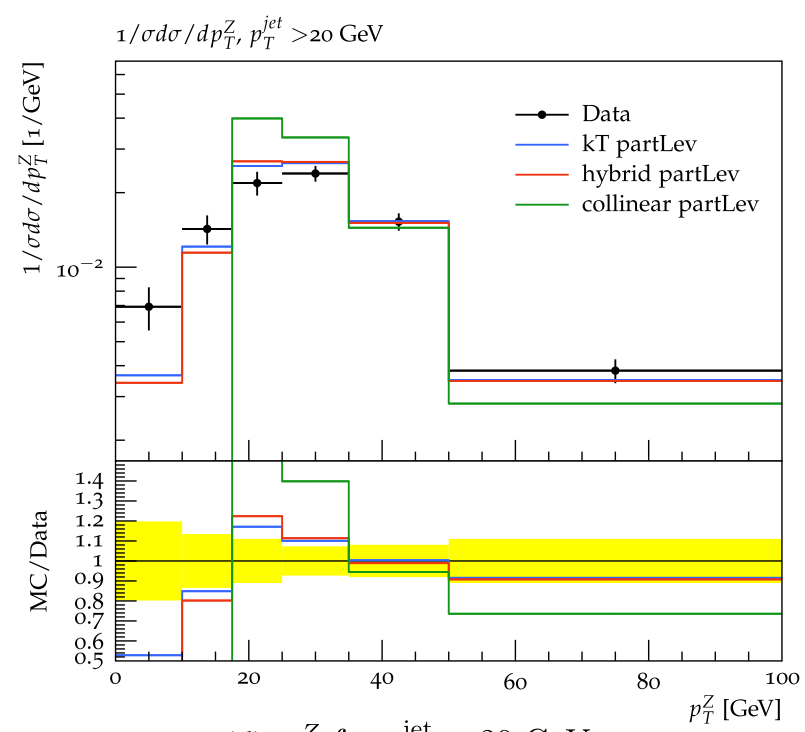

(d) $p_{T}^{Z}$ for $p_{T}^{\text {jet }}>20 \mathrm{GeV}$

FIG. 3. Comparison of $\Delta \phi$ and $p_{T}^{Z}$ distributions calculated on parton level (without any showers) and the LHCb measurements [13]. 2 (a), 2(c) for $p_{T}^{\text {jet }}>10 \mathrm{GeV}$ and 2(b), 2(d) for $p_{T}^{\text {jet }}>20 \mathrm{GeV}$. The factorization/renormalization scale $\mu=\sqrt{m_{Z}^{2}+\left(p_{T}^{\text {jet }}\right)^{2}}$ is used for $k_{T^{-}}$ factorization (two off-shell initial partons), hybrid factorization (one off-shell and one on-shell initial parton), and collinear factorization (two on-shell partons).

similar to the results at the parton level, the $k_{T}$-factorization and hybrid factorization approaches give very similar results. On the other hand, we see a substantial change in the case of predictions from collinear factorization, which now is also quite similar to the other two predictions: the $\Delta \phi$ distribution in the collinear case is no longer zero and it gives a rather good description of the data. Note here that the $\Delta \phi$ distribution is fully generated by the TMD parton densities (and the corresponding TMD shower). From a kinematic point of view this mimics the features of the off-shell calculation, where the initial transverse momenta are included from the beginning. It also points to the observation that initial state transverse momenta are perhaps more important than the off-shellness in the matrix elements: the off-shell matrix elements would contribute a dynamical correction, while the kinematics are driven by including the transverse momenta from the TMD parton densities. While calculations obtained in $k_{T}$ and hybrid factorization give essentially the same results, a difference in results from collinear factorization with parton shower is observed. The parton densities and the parton showers are the same for all calculations, and the difference comes entirely from the different matrix elements used (off shell versus on shell). 


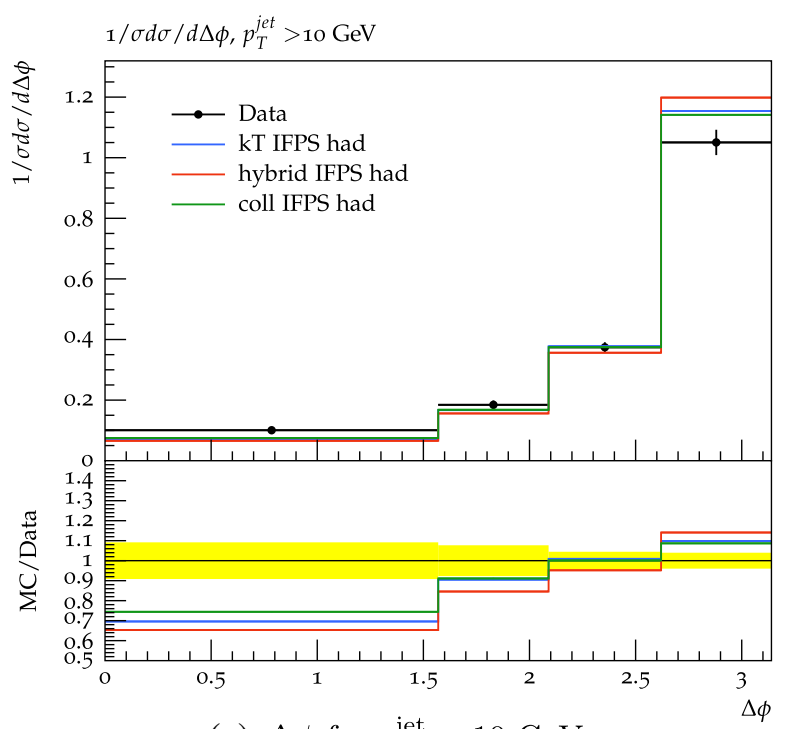

(a) $\Delta \phi$ for $p_{T}^{\text {jet }}>10 \mathrm{GeV}$

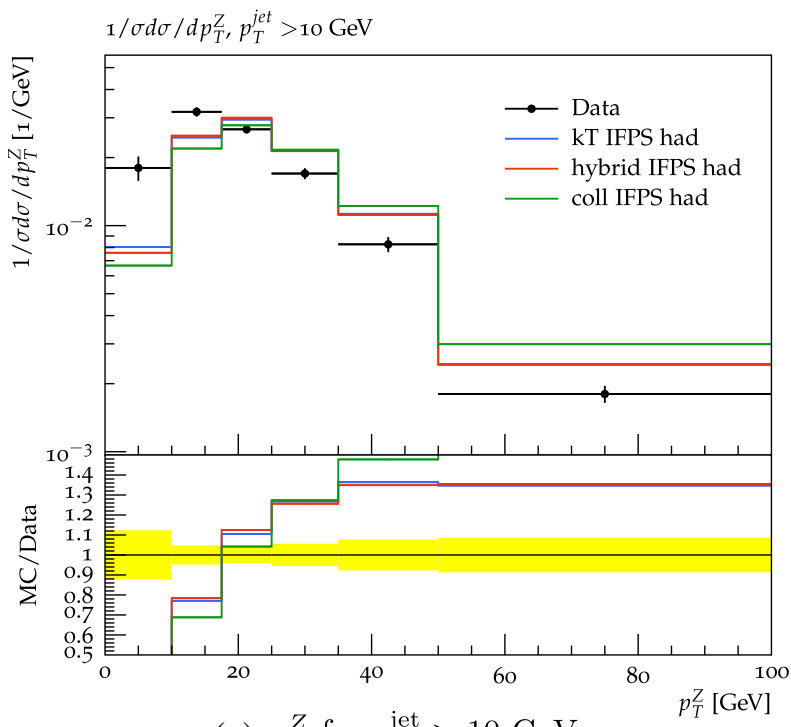

(c) $p_{T}^{Z}$ for $p_{T}^{\text {jet }}>10 \mathrm{GeV}$

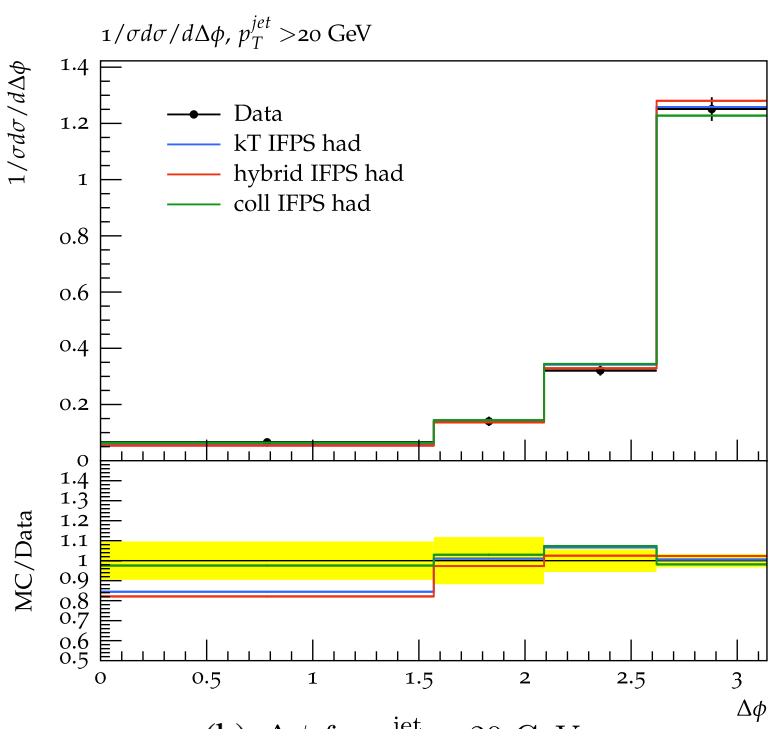

(b) $\Delta \phi$ for $p_{T}^{\text {jet }}>20 \mathrm{GeV}$

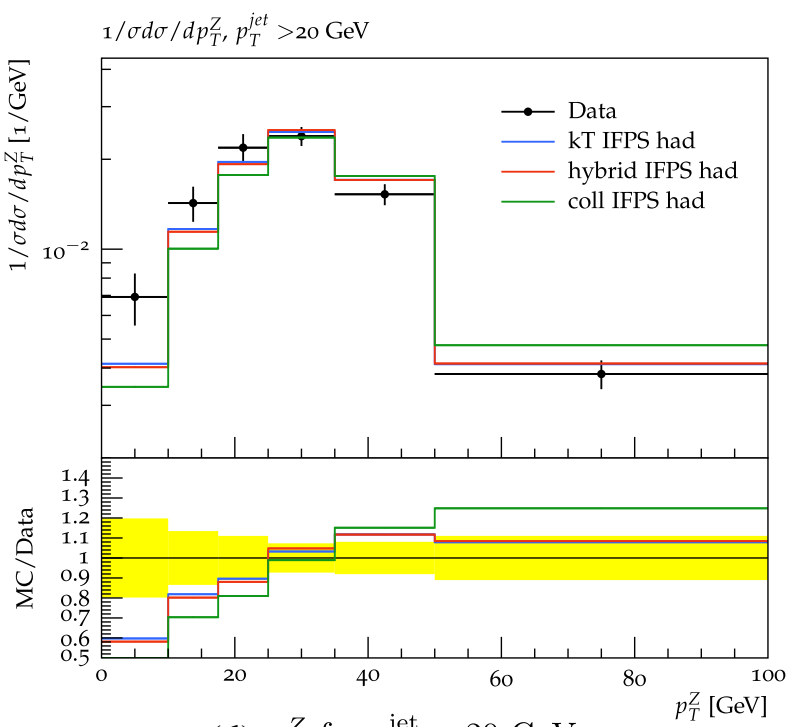

(d) $p_{T}^{Z}$ for $p_{T}^{\text {jet }}>20 \mathrm{GeV}$

FIG. 4. Comparison of $\Delta \phi$ and $p_{T}^{Z}$ distributions calculated including initial and final state showers as well as hadronization, using factorization/renormalization scale $\mu=\sqrt{m_{Z}^{2}+\left(p_{T}^{\text {jet }}\right)^{2}}$ within: $k_{T}$-factorization (two off-shell initial partons), hybrid factorization (one off-shell and one on-shell initial parton), and collinear factorization (two on-shell partons). Compared to the LHCb measurements [13].

The $\Delta \phi$ distributions are rather well described by all approaches, while in the $p_{T}^{Z}$ distribution, all approaches predict a too small cross section at small $p_{T}^{Z}$, the $k_{T^{-}}$ factorization and hybrid factorization are slightly better than the collinear calculation with TMD showers included. We have checked that changing the scale $\mu$ gives similar effects as observed without TMD showers.

Finally, in Fig. 5 we compare $k_{T}$-factorization predictions with parton showers and hadronization (including scale variations by a factor of 2) with a collinear NLO calculation of $Z$ boson and two jets performed in POWHEG (with the multiscale improved NLO method $[33,34]$ ), using the HERAPDF20_NLO collinear PDFs [28] and the pythia 8 tune CUETP8M1 [35]. We show results with and without multiparton interactions (MPIs) in order to see whether there is any numerical correspondence between the $k_{T^{-}}$ factorization calculation and collinear calculation assuming no MPI contribution in the latter. The assumption is needed since so far the formulation of MPI for $k_{T}$-factorization has not been obtained.

The measured $\Delta \phi$ distribution is well described by the NLO collinear calculation [Figs. 5(a) and 5(b)], when MPI is included. Similarly, the POWHEG calculation agrees rather well with the measured $p_{T}^{Z}$ distribution [Figs. 5(c) 


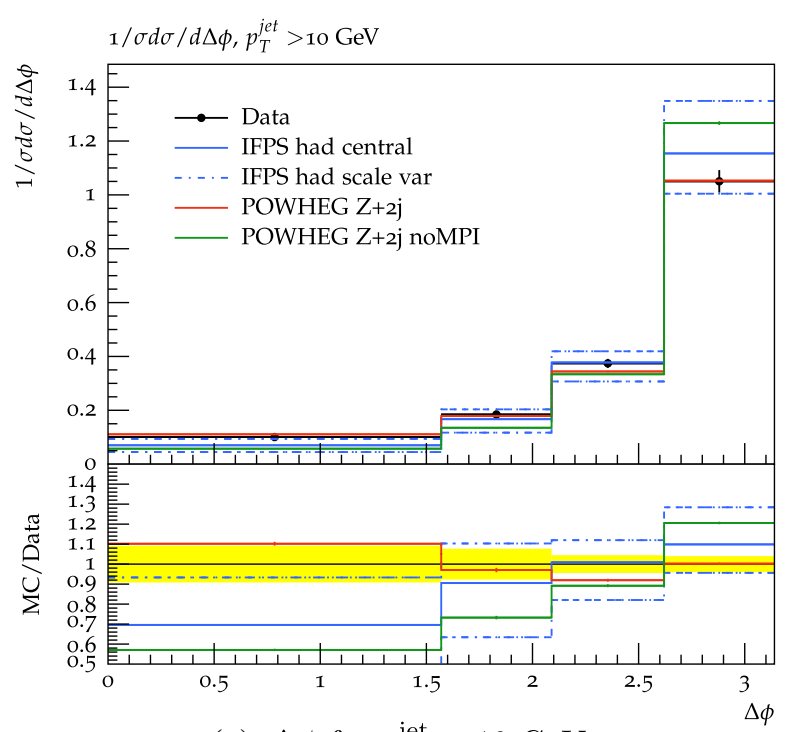

(a) $\Delta \phi$ for $p_{T}^{\text {jet }}>10 \mathrm{GeV}$

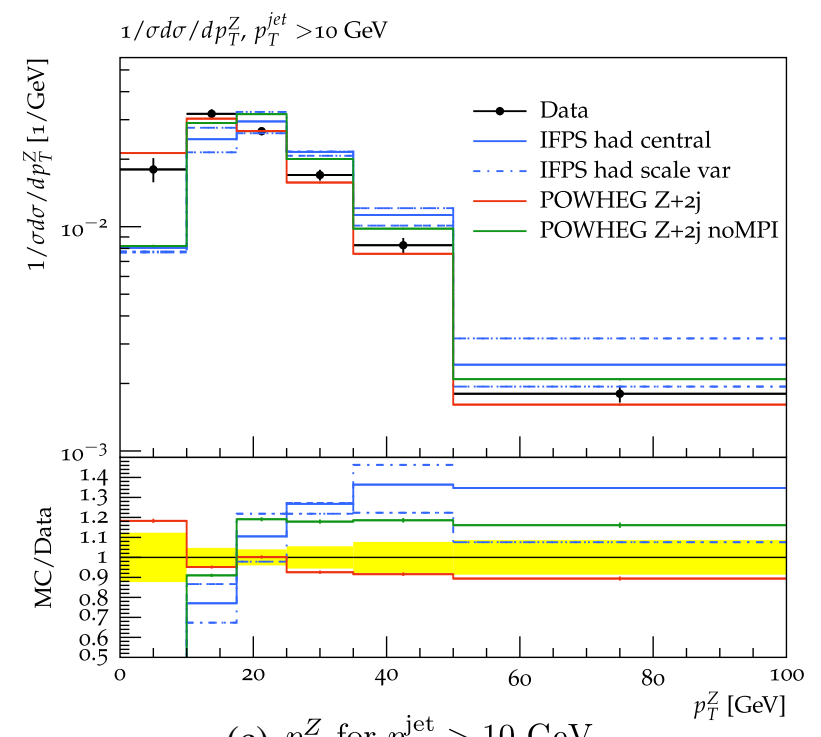

(c) $p_{T}^{Z}$ for $p_{T}^{\text {jet }}>10 \mathrm{GeV}$

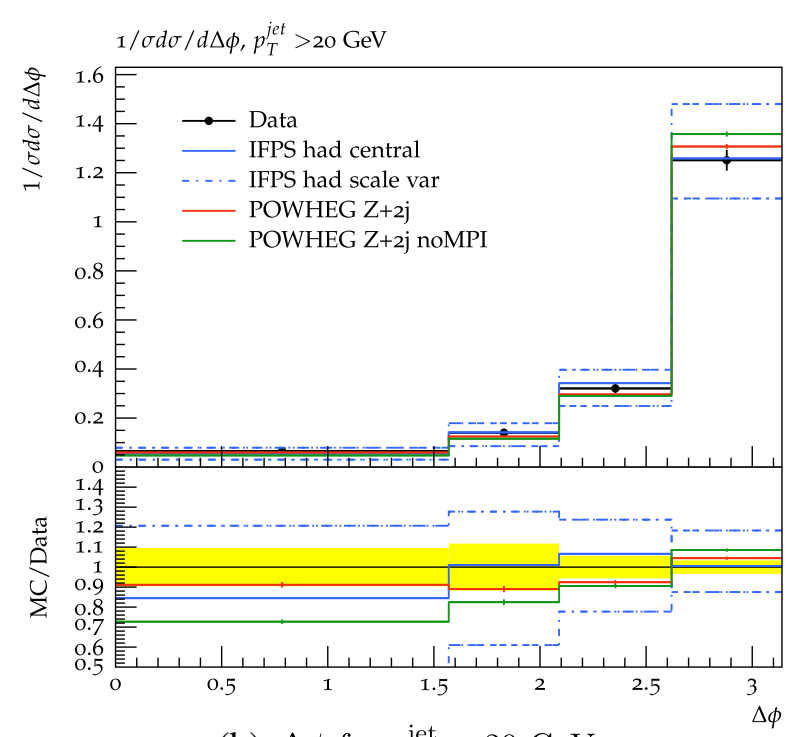

(b) $\Delta \phi$ for $p_{T}^{\text {jet }}>20 \mathrm{GeV}$

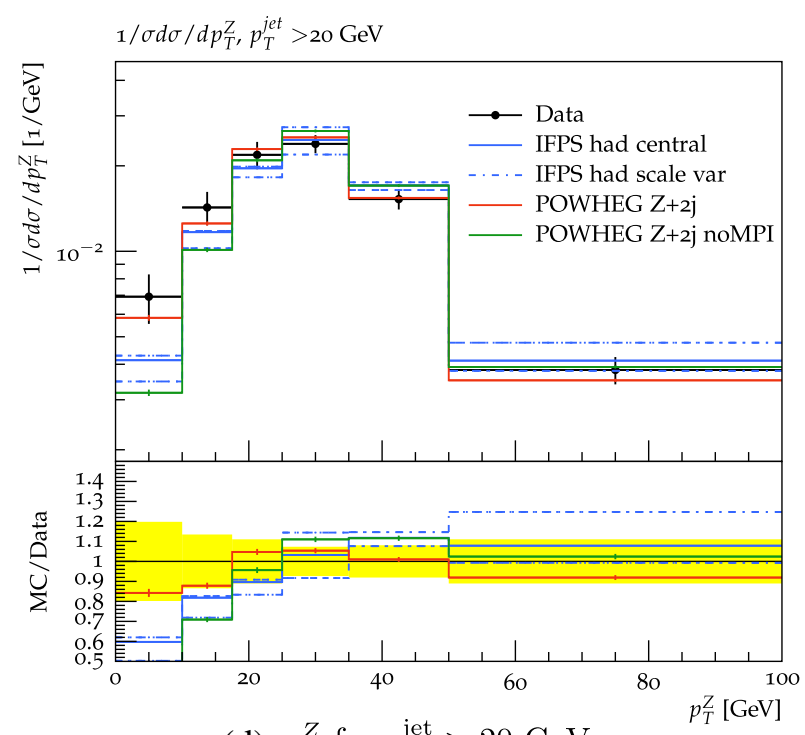

(d) $p_{T}^{Z}$ for $p_{T}^{\text {jet }}>20 \mathrm{GeV}$

FIG. 5. Scale variation for $\Delta \phi$ and $p_{T}^{Z}$ distributions calculated including initial and final state showers as well as hadronization using factorization/renormalization scale $\mu=\sqrt{m_{Z}^{2}+\left(p_{T}^{\text {jet }}\right)^{2}}$ compared with POwHEG results for $Z+2$ jet production. Comparison to the LHCb measurements [13].

and 5(d)], if MPI is included, in particular at small $p_{T}^{Z}$, below the $p_{T}^{\text {jet }}$ cutoff. It is interesting to note that at low $p_{T}^{Z}$ the description of the measurement is significantly improved when MPI is included, meaning that some of the low $p_{T}$ jets come from MPI. If the contribution from MPI is switched off, then the predictions calculated with collinear NLO $Z+2$ jet and with off-shell matrix elements in LO $k_{T}$-factorization agree rather well. This observation confirms that the distributions using off-shell matrix elements are similar to the ones obtained by a collinear NLO calculation.

The differential distributions measured by $\mathrm{LHCb}$ are normalized to the total cross section which means we could not judge the normalization of our predictions. In order to do it in Table I we show a comparison of the measured total cross section with the different predictions. All LO predictions [using $\alpha_{s}\left(m_{Z}\right)=0.118$ ] are significantly smaller than the measurements. This is partly caused by the use of NLO PDFs and two-loop $\alpha_{s}$. We have verified that using consistently LO PDFs and strong coupling (we used HERAPDF20_LO_EIG PDF set) increases the cross section and brings it closer to the measurement but it does not explain the whole difference.

Additionally, Table I shows us also the importance of the MPI for the total cross section. 
TABLE I. Total cross section for $Z+$ jet as measured by LHCb [13] and predicted by the corresponding calculations in $k_{T}$, hybrid, and collinear factorization. All the calculations include showering and hadronization and were performed using the scale $\mu=\sqrt{m_{Z}^{2}+\left(p_{T}^{\text {jet }}\right)^{2}}$. The uncertainties of the theoretical predictions are numerical integration errors.

\begin{tabular}{lccccrr}
\hline \hline$p_{T}^{\text {jet }}(\mathrm{GeV})$ & \multicolumn{1}{c}{ LHCb } & $k_{T}$ & Hybrid & Coll. (LO) & Coll. (NLO) no MPI & Coll. (NLO) with MPI \\
\hline 20 & $6.3 \pm 0.55$ & $3.6 \pm 1 \times 10^{-5}$ & $3.9 \pm 2 \times 10^{-5}$ & $4.6 \pm 4 \times 10^{-5}$ & $5.2 \pm 0.04$ & $5.5 \pm 0.4$ \\
10 & $16.0 \pm 1.36$ & $7.5 \pm 2 \times 10^{-5}$ & $7.9 \pm 4 \times 10^{-5}$ & $9.1 \pm 7 \times 10^{-5}$ & $11.4 \pm 0.09$ & $15.5 \pm 1.2$ \\
\hline \hline
\end{tabular}

\section{Correlations}

In this section we will analyze correlations between longitudinal and transverse components of the initial state partons.

In Fig. 6 the correlations between longitudinal momentum fractions of the initial state partons $x_{1}$ and $x_{2}$ are shown for both factorization schemes [high energy (HE) and hybrid], for the region $20<p_{T}^{Z}<30 \mathrm{GeV}$ (results for other ranges of $p_{T}^{Z}$ are similar). In the region of forward $Z$ production, as defined by the $\mathrm{LHCb}$ measurement, one of the partons has a small longitudinal component $\left(x_{2} \sim 10^{-3}\right)$, whereas the second parton has rather large values $x_{1} \sim 0.3$. In the hybrid approach, the initial off-shell parton is the one at low value of $x$, namely $x_{2}$.

In Fig. 7 the correlations between the transverse momenta of the initial state partons are shown for the case

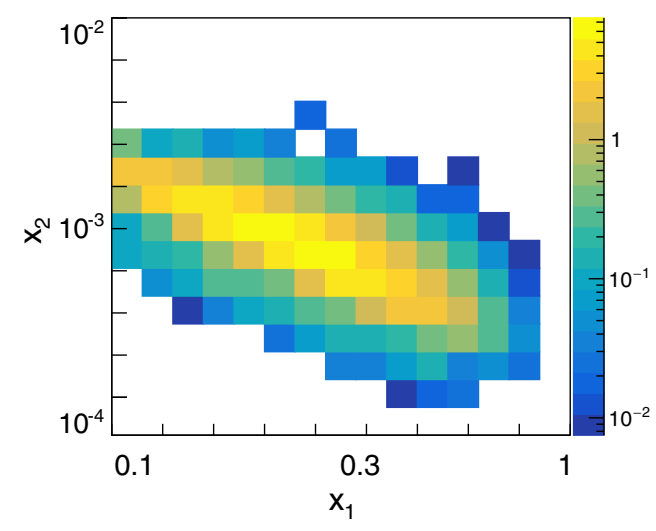

(a) $k_{T}$-approach

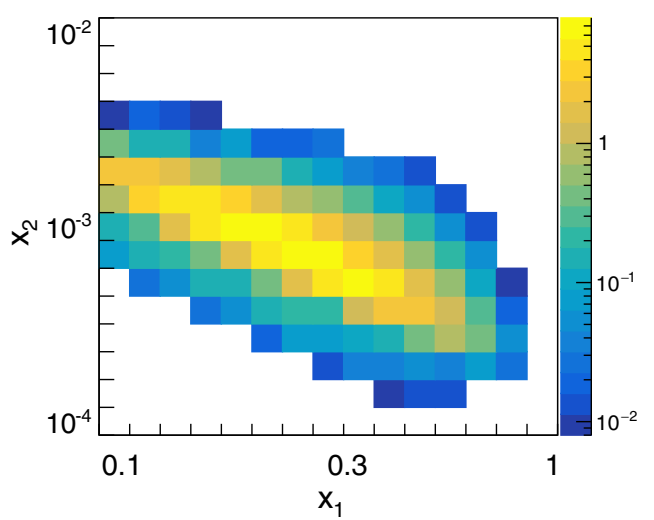

(b) hybrid-approach

FIG. 6. Correlation between longitudinal momentum factions $x$ of the initial state partons for a selected bin of $Z$-boson transverse momentum: $20<p_{T}^{Z}<30 \mathrm{GeV}$. Distributions for other bins in $p_{T}^{Z}$ look similar.

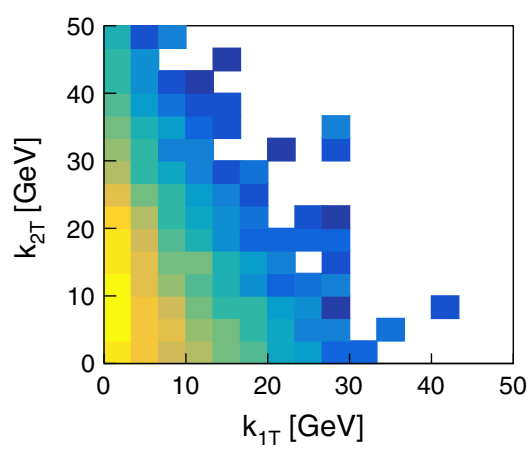

(a) $0<p_{T}^{Z}<10 \mathrm{GeV}$

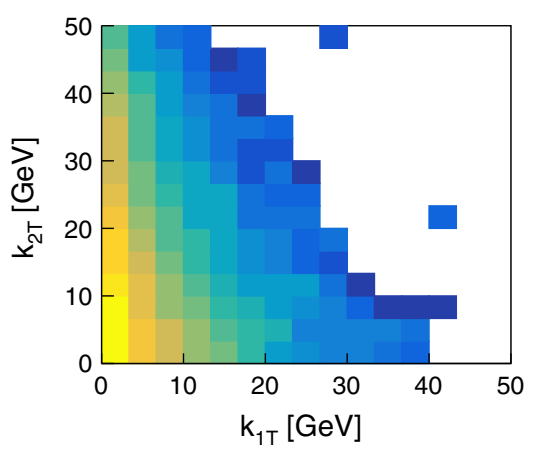

(b) $20<p_{T}^{Z}<30 \mathrm{GeV}$

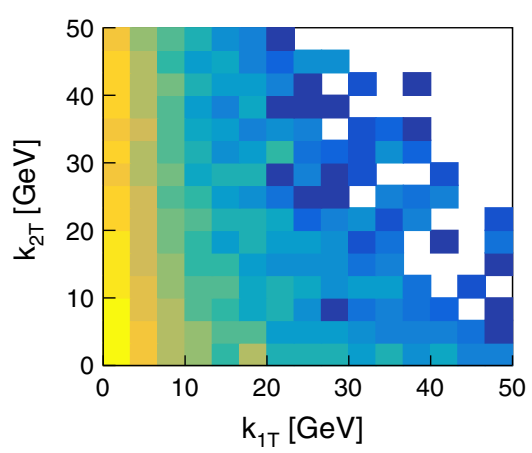

(c) $50<p_{T}^{Z}<60 \mathrm{GeV}$

FIG. 7. Correlation between transverse momenta of the initial state partons $k_{1 T}$ (with large $x$ value) and $k_{2 T}$ (with low $x$ value) for selected bins of $Z$-boson transverse momentum for the $k_{T}$-factorization approach. 


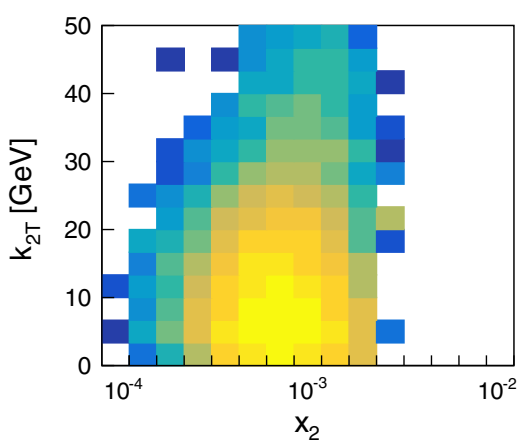

(a) $0<p_{T}^{Z}<10 \mathrm{GeV}$

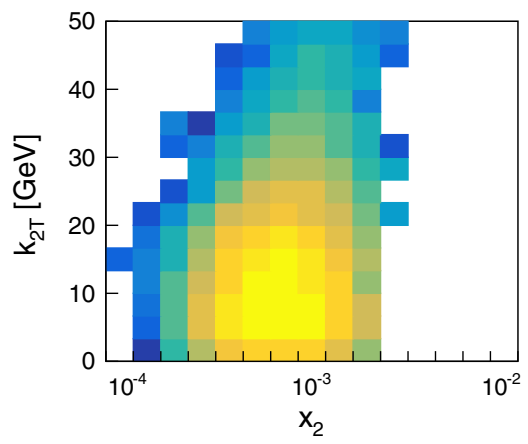

(d) $0<p_{T}^{Z}<10 \mathrm{GeV}$

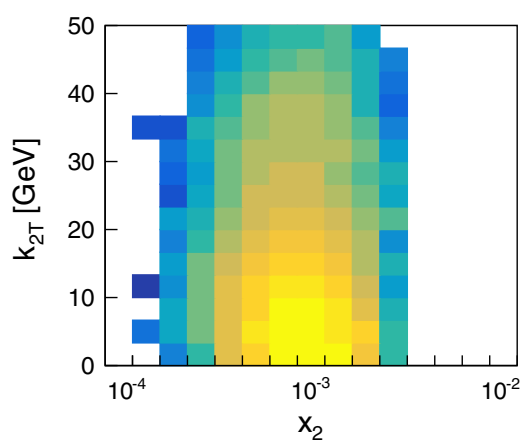

(b) $20<p_{T}^{Z}<30 \mathrm{GeV}$

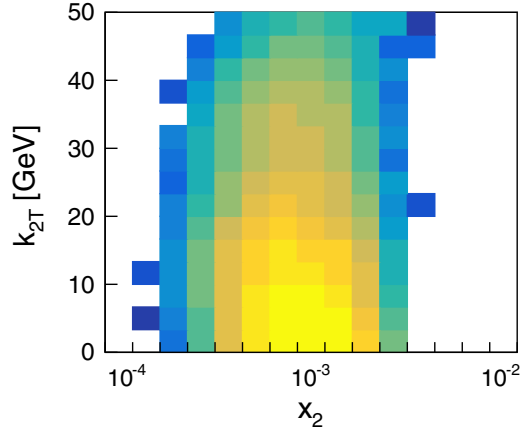

(e) $20<p_{T}^{Z}<30 \mathrm{GeV}$

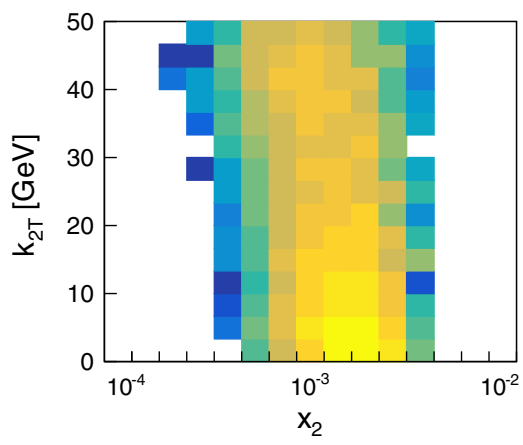

(c) $50<p_{T}^{Z}<60 \mathrm{GeV}$

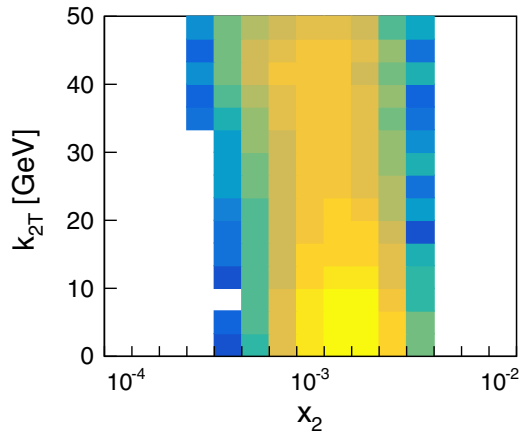

(f) $50<p_{T}^{Z}<60 \mathrm{GeV}$

FIG. 8. Correlation between longitudinal momentum faction $x_{2}$ and transverse momentum of the second initial state parton $k_{2 T}$ (low longitudinal momentum fraction) for selected bins of the Z-boson transverse momentum. The upper row displays results for $k_{T^{-}}$ factorization, lower row for the hybrid approach.

of $k_{T}$-factorization (in the hybrid approach the $k_{1 T}$ momentum is zero). ${ }^{2}$ One of the transverse momenta, $k_{1 T}$ (large $\left.x_{1}\right)$, is small $\left(k_{1 T} \lesssim 5 \mathrm{GeV}\right)$, while the other one, $k_{2 T}$ (low $x_{2}$ ), has a much broader distribution. The average transverse momentum $k_{2 T}$ increases for increasing $p_{Z}^{Z}$, as shown in Fig. 7, while the transverse momentum of the other parton stays very small. This observation explains why the predictions in HE (two off-shell partons) and hybrid factorization (only one off-shell parton) give very similar results.

In Fig. 8 the correlation between the longitudinal and the transverse momentum of the low energetic parton is shown, for both $k_{T}$-factorization and hybrid factorization approaches. With increasing transverse momentum of the $Z$ boson, the longitudinal momentum $x_{2}$ also increases. Both factorization approaches show very similar correlations between $x_{2}$ and $k_{2 T}$ as a function of $p_{T}^{Z}$, confirming again the equivalence of $k_{T}$-factorization and hybrid factorization results in the region of forward $Z$ production.

\section{CONCLUSION}

We presented calculations of $Z+1$ jet final states including transverse momenta of the initial state partons

\footnotetext{
${ }^{2}$ The scale for all the color-map plots is the same as in Fig. 6.
}

and compared the predictions with measurements of the $\mathrm{LHCb}$ experiment. The calculations were performed using the KaTie parton-level event generator together with initial state parton showers implemented in a new version of CASCADE. We applied consistently the PB TMD parton densities together with two-loop $\alpha_{s}\left(m_{Z}\right)=0.118$.

The predictions obtained in $k_{T}$-factorization and hybrid factorization agree very well with each other for the forward $Z$ production pointing towards effective equivalence of the two approaches in the forward region. The predictions obtained in collinear factorization at leading order for $Z+1$ jet, supplemented with PB TMD parton densities and corresponding parton shower show differences to predictions obtained in $k_{T}$-factorization, which come from the different matrix elements used. A comparison of prediction obtained in $k_{T}$-factorization and a collinear NLO calculation of $Z+2$ jet supplemented with standard parton showers shows very good agreement. The description of the experimental measurement especially at very small $p_{T}^{Z}$ is significantly improved when contributions from multiparton interactions are included.

The predictions obtained in $k_{T}$-factorization (as well as in hybrid factorization) agree rather well with the measurements of the LHCb Collaboration. Differences are observed in the region of small $p_{T}^{Z}$, where the predictions depend significantly on the treatment of multiparton 
interactions, which are not yet included in the calculations with $k_{T}$-factorization.

We presented a first consistent comparison of calculations in different factorization approaches, and illustrated the features and advantages of using off-shell matrix elements obtained in $k_{T}$-factorization.

\section{ACKNOWLEDGMENTS}

K. K. acknowledges the support of Narodowe Centrum Nauki with Grant No. DEC-2017/27/B/ST2/01985. M. D.,
A. v. H., and A. K. acknowledge the support by the FWOPAS VS.070.16N research grant. A. v. H. was also partially supported by a grant of National Science Center, Poland, Grant No. 2015/17/B/ST2/01838. M. S. is supported by the Israeli Science Foundation through Grant No. 1635/16, by BSF Grants No. 2012124 and No. 2014707, by COST Action No. CA15213 THOR, and by a Kreitman fellowship by the Ben Gurion University of the Negev. H. J. thanks the Polish Science Foundation for the Humboldt Research fellowship during which part of this work was completed.
[1] F. Hautmann, M. Hentschinski, and H. Jung, Forward $Z$-boson production and the unintegrated sea quark density, Nucl. Phys. B865, 54 (2012).

[2] L. Motyka, M. Sadzikowski, and T. Stebel, Twist expansion of Drell-Yan structure functions in color dipole approach, J. High Energy Phys. 05 (2015) 087.

[3] A. van Hameren, P. Kotko, and K. Kutak, Resummation effects in the forward production of $Z_{0}+$ jet at the LHC, Phys. Rev. D 92, 054007 (2015).

[4] W. Schfer and A. Szczurek, Drell-Yan production at forward rapidities: A hybrid factorization approach, in Proceedings of the 24th Low-x Meeting (Low-x 2016), Gyongyos, Hungary, 2016.

[5] F. G. Celiberto, D. Gordo Gmez, and A. Sabio Vera, Forward Drell-Yan production at the LHC in the BFKL formalism with collinear corrections, Phys. Lett. B 786, 201 (2018).

[6] R. Angeles-Martinez et al., Transverse momentum dependent (TMD) parton distribution functions: Status and prospects, Acta Phys. Pol. B 46, 2501 (2015).

[7] M. Deak, F. Hautmann, H. Jung, and K. Kutak, Forwardcentral jet correlations at the Large Hadron Collider, arXiv:1012.6037.

[8] S. Catani, M. Ciafaloni, and F. Hautmann, High-energy factorization and small $x$ heavy flavor production, Nucl. Phys. B366, 135 (1991).

[9] A. van Hameren, KaTie: For parton-level event generation with $k_{T}$-dependent initial states, Comput. Phys. Commun. 224, 371 (2018).

[10] H. Jung, S. Baranov, M. Deak, A. Grebenyuk, F. Hautmann et al., The CCFM Monte Carlo generator CASCADE version 2.2.03, Eur. Phys. J. C 70, 1237 (2010).

[11] M. Bury, A. van Hameren, H. Jung, K. Kutak, S. Sapeta, and M. Serino, Calculations with off-shell matrix elements, TMD parton densities and TMD parton showers, Eur. Phys. J. C 78, 137 (2018).

[12] A. Dumitru, A. Hayashigaki, and J. Jalilian-Marian, The color glass condensate and hadron production in the forward region, Nucl. Phys. A765, 464 (2006).

[13] R. Aaij et al. (LHCb Collaboration), Study of forward $Z+$ jet production in $p p$ collisions at $\sqrt{s}=7 \mathrm{TeV}$, J. High Energy Phys. 01 (2014) 033.
[14] P. Kotko, K. Kutak, C. Marquet, E. Petreska, S. Sapeta, and A. van Hameren, Improved TMD factorization for forward dijet production in dilute-dense hadronic collisions, J. High Energy Phys. 09 (2015) 106.

[15] A. van Hameren, P. Kotko, K. Kutak, C. Marquet, E. Petreska, and S. Sapeta, Forward di-jet production in $p+$ $\mathrm{Pb}$ collisions in the small- $x$ improved TMD factorization framework, J. High Energy Phys. 12 (2016) 034.

[16] A. van Hameren, P. Kotko, and K. Kutak, Multi-gluon helicity amplitudes with one off-shell leg within high energy factorization, J. High Energy Phys. 12 (2012) 029.

[17] A. van Hameren, P. Kotko, and K. Kutak, Helicity amplitudes for high-energy scattering, J. High Energy Phys. 01 (2013) 078.

[18] E. N. Antonov, L. N. Lipatov, E. A. Kuraev, and I. O. Cherednikov, Feynman rules for effective Regge action, Nucl. Phys. B721, 111 (2005).

[19] M. A. Kimber, A. D. Martin, and M. G. Ryskin, Unintegrated parton distributions, Phys. Rev. D 63, 114027 (2001).

[20] A. D. Martin, M. G. Ryskin, and G. Watt, NLO prescription for unintegrated parton distributions, Eur. Phys. J. C 66, 163 (2010).

[21] F. Hautmann, H. Jung, A. Lelek, V. Radescu, and R. Zlebcik, Collinear and TMD quark and gluon densities from parton branching solution of QCD evolution equations, J. High Energy Phys. 01 (2018) 070.

[22] F. Hautmann, H. Jung, A. Lelek, V. Radescu, and R. Zlebcik, Soft-gluon resolution scale in QCD evolution equations, Phys. Lett. B 772, 446 (2017).

[23] A. Bermudez Martinez, P. Connor, F. Hautmann, H. Jung, A. Lelek, V. Radescu, and R. Zlebcik, Collinear and TMD parton densities from fits to precision DIS measurements in the parton branching method, Phys. Rev. D 99, 074008 (2019).

[24] T. Sjöstrand, S. Mrenna, and P. Skands, pythia 6.4 physics and manual, J. High Energy Phys. 05 (2006) 026.

[25] B. Jager, S. Schneider, and G. Zanderighi, Next-toleading order QCD corrections to electroweak Zjj production in the POWHEG BOX, J. High Energy Phys. 09 (2012) 083. 
[26] T. Sjöstrand, S. Ask, J. R. Christiansen, R. Corke, N. Desai, P. Ilten, S. Mrenna, S. Prestel, C. O. Rasmussen, and P. Z. Skands, An introduction to pythia 8.2, Comput. Phys. Commun. 191, 159 (2015).

[27] F. Hautmann, H. Jung, M. Krämer, P. J. Mulders, E. R. Nocera, T. C. Rogers, and A. Signori, TMDlib and TMDplotter: Library and plotting tools for transversemomentum-dependent parton distributions, Eur. Phys. J. C 74, 3220 (2014).

[28] H. Abramowicz et al. (ZEUS and H1 Collaborations), Combination of measurements of inclusive deep inelastic $e^{ \pm} p$ scattering cross sections and QCD analysis of HERA data, Eur. Phys. J. C 75, 580 (2015).

[29] A. Buckley, J. Butterworth, L. Lonnblad, D. Grellscheid, H. Hoeth, J. Monk, H. Schulz, and F. Siegert, RIVET user manual, Comput. Phys. Commun. 184, 2803 (2013).
[30] H. Jung, $k_{t}$-factorization and CCFM: The solution for describing the hadronic final states everywhere?, Mod. Phys. Lett. A 19, 1 (2004).

[31] H. Jung, The CCFM Monte Carlo generator CASCADE, Comput. Phys. Commun. 143, 100 (2002).

[32] J. Alwall et al., A standard format for Les Houches event files, Comput. Phys. Commun. 176, 300 (2007).

[33] K. Hamilton, P. Nason, and G. Zanderighi, MINLO: Multiscale improved NLO, J. High Energy Phys. 10 (2012) 155.

[34] K. Hamilton, P. Nason, C. Oleari, and G. Zanderighi, Merging $\mathrm{H} / \mathrm{W} / \mathrm{Z}+0$ and 1 jet at NLO with no merging scale: A path to parton shower + NNLO matching, J. High Energy Phys. 05 (2013) 082.

[35] V. Khachatryan et al. (CMS Collaboration), Event generator tunes obtained from underlying event and multiparton scattering measurements, Eur. Phys. J. C 76, 155 (2016). 